\title{
Erratum to "Effect of Concentric Annular Gap Flow on Wall Shear Stress of Stationary Cylinder Pipe Vehicle under Different Reynolds Numbers"
}

\author{
Xiaomeng Jia, ${ }^{1}$ Xihuan Sun $\mathbb{D}^{1,2}$ and Jiaorong Song ${ }^{1}$ \\ ${ }^{1}$ Country. College of Water Resource Science and Engineering, Taiyuan University of Technology, Taiyuan, China \\ ${ }^{2}$ Jin Zhong University, Jinzhong, China \\ Correspondence should be addressed to Xihuan Sun; sunxihuan@tyut.edu.cn
}

Received 15 October 2020; Accepted 15 October 2020; Published 24 October 2020

Copyright ( 2020 Xiaomeng Jia et al. This is an open access article distributed under the Creative Commons Attribution License, which permits unrestricted use, distribution, and reproduction in any medium, provided the original work is properly cited.

In the article titled "Effect of Concentric Annular Gap Flow on Wall Shear Stress of Stationary Cylinder Pipe Vehicle under Different Reynolds Numbers" [1], there was an error in equations (8) and (9). The corrected equations are shown as follows:

$$
\begin{aligned}
& F_{f}=\mu F_{n 1}=\mu \frac{G-F_{c}}{2}, \\
& F_{\tau}=\frac{\lambda_{c}}{8} \rho\left(v_{a}-v_{c}\right)^{2} A^{\prime},
\end{aligned}
$$

where $\lambda_{c}$ represents the flow resistance coefficient, which is related to the material of the inner wall of the pipe and the physical properties of the fluid; $v_{a}$ represents the velocity of the annular gap flow; and $v_{c}$ represents the velocity of water in the pipe. $A^{\prime}$ represents the lateral area of the piped vehicle.

The error was introduced during the production process of the article, and Hindawi apologises for causing this error in the article.

\section{References}

[1] X. Jia, X. Sun, and J. Song, "Effect of concentric annular gap flow on wall shear stress of stationary cylinder pipe vehicle under different Reynolds numbers," Mathematical Problems in Engineering, vol. 2020, Article ID 1253652, 19 pages, 2020. 